\title{
WAVELET TRANSFORM BASED ON QRS DETECTION USING DIODIC ALGORITHM
}

\author{
Basavaraj Biradar $^{1}$, Geeta Patil ${ }^{2}$, Shweta Telgane ${ }^{3}$, S G Hiremath ${ }^{4}$ \\ ${ }^{I}$ Department of E \& CE, Udaygiri College of engineering Udgir- 413517. Maharashtra, India \\ ${ }^{2}$ Department of E \& CE, BKEC Basavakalan, Karnataka \\ ${ }^{3}$ Department of E \& CE, GNDEC Bidar: 585401. Karnataka, India \\ ${ }^{4}$ Department of E \& CE, East West Institute of Technology Banglore-560091
}

\begin{abstract}
ECG system is the backbone of cardiac disease detection and cure procedure. In order to enrich the technology and versatility of ECG signal a new approach used in detection of QRS complex in ECG signals. The proposed system is based on dyadic wavelet transform $D_{y} W T$ which prevent time varying $Q R S$ complex as well as noise. The QRS complex computes heart rate between two $R$ waves by considering the spectral characteristics of ECG signal. We propose the spline wavelet which is suitable for detection of QRS complex. In order to preserve the standards, the ECG specimen signals are taken from American heart association (AHA) database. Seventy hours of data have been considering for the analysis. We compare the performance of DyWT-based QRS detector with detectors based on Okada, Hamilton-Tompkins, and multiplication of the backward difference algorithms. After comparing these algorithms, the $D_{y} W T$ detector gives better quality performance in all situations. Hence we consider $D_{y} W T$ is standard techniques among all above said algorithm. Then QRS complexes will be detected and each complex will be used to find the peaks of the individual waves like, $P$ and $T$, and their deviations.
\end{abstract}

Keywords: Electrocardiogram (ECG), Dyadic wavelet transforms ( $\left.D_{y} W T\right)$, American heart association (AHA), and QRS detection. - ***

\section{INTRODUCTION OF QRS COMPLEX DETECTOR}

The abbreviation ECG stand for electrocardiogram is a graphic record of the direction and magnitude of the electrical activity that is generated by depolarization and repolarization of the atria and ventricles. But even though the QRS complex is the dominant feature of the ECG signal and detection of QRS complex in ECG signal very difficult task. Because it has a time varying structure and is focused on physiological changes in patients and ECG signal they are not always the strongest signal due to noise. Cardiac patients need to know direct visual monitoring of ECGs, so this is tough task by human being because whose monotony increases the loss of biological information. The great efforts by biomedical research centers have been made to developed analog and digital systems for ECG analysis. Computer based ECG analysis system have proved to be more efficient, having made possible rapid retrieval of data for storage and techniques of data arrangement whose clinical utility is evident. In Fig.1.1 describe the ECG signals $P$ interval-QRS interval and $T$ interval detection of interval in ECG signal is more complex. Purposely QRS wave is used to detect arrhythmias and identify problems in regularity of heart rate. QRS complex in ECG signal has been changing with time and corrupted with noise therefore complicated to detect the $\mathrm{R}$ wave which is the highest point of the QRS complex. Sometimes, in an ECG signal, QRS complexes may not always be the significant waves because they change their structure with respect to time for different conditions, so that consider always be the strongest signal interval in an ECG signal. The identification of QRS complex is tough task because $\mathrm{P}$ or $\mathrm{T}$ wave have similar characteristics as QRS and ECG signal can be affected and degraded by other sources such as noise in a clinical environment like patient condition, baseli Wandering due to respiration, patient movement, interference of the input power supply, contraction and twitching of the muscles and weak contact of the ECG electrodes. Therefore, it is essential for the QRS detector to avoid the noise.

The major important feature of ECG to compute accurate and quick method for and several related arrhythmias such as Tachycardia, Bradycardia and Heart Rate Variation; it is also necessary for further processing of the signal in order to detect abnormal beats. 


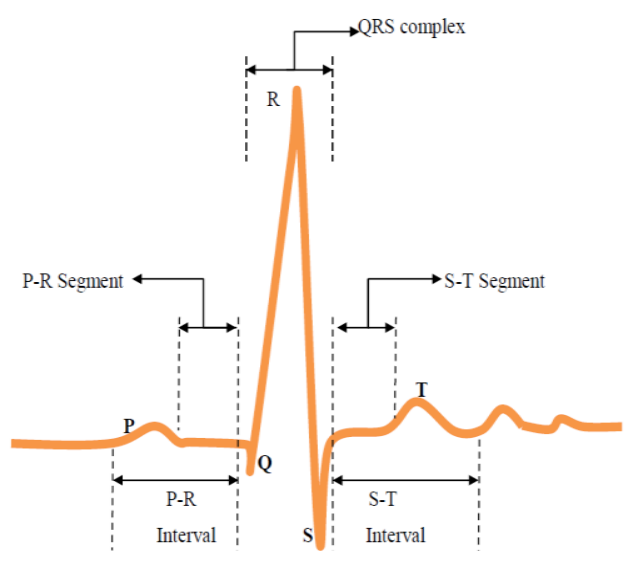

Fig 1 ECG Waveform and QRS complex interval

\subsection{Literature Review}

Fraden and Neuman (1980) developed a QRS detection scheme where a threshold was calculated as a fraction of the peak value of the ECG. In 1985, Pan and Tompkin proposed an algorithm to recognize QRS complex in which they analyzed the positions and magnitudes of sharp waves and used a special digital band pass filter (BPF) to reduce the false detection of ECG signals. Also in 1997 Ruha et al filtered the ECG signal with a matched filter to suppress the $\mathrm{P}$ and $\mathrm{T}$ waves and noise first, then the QRS complexes were enhanced by passing through a nonlinear transformation, at last, the QRS complexese location were determined. Li et al (1995) who proposed a method based on finding the modulus maxima larger than a threshold obtained from the pre-processing of preselected initial beats. In $\mathrm{Li}$ et al"s method, the threshold is updated during the analysis to obtain a better performance. This method has a post-processing phase in which redundant $\mathrm{R}$ waves or noise peaks are removed. The algorithm achieves a good performance with a reported sensitivity of $99.90 \%$ and positive prediction value of $99.94 \%$ when tested on the MIT/BIH database. Wavelet based QRS detection are also widely examined (1995; Li et al.,1993; Sahambi et al.,1997; Mahmoodabadi et al., 2005; Sasikala et al 2010; Bsoul et al., 2009). With the wavelet based analysis, each QRS complex corresponds to a couple of maximum and minimum in wavelet transform. Using different scales in the analysis in time-and frequency-domain, the signals are divided into different response clusters which represent different frequency components of the ECG. Kozakevicius et al (1988) utilized orthogonal wavelets to filter and analyze ECG signals. They used compactly supported wavelets associated to the statistical Stein"s Unbiased Risk Estimator in order to obtain an adaptive thresholding strategy to filter ECG signals and then they analyzed the filtered signals by using the Haar wavelet transform in order to detect the positions of the occurrence of the QRS complex during the period of analysis.
Legarreta et al (2005) have extended the work of $\mathrm{Li}$ et al and Kadambe et al, utilizing the continuous wavelet transform. Their CWT-based algorithm affords high time-frequency resolution which provides a better definition of the QRS modulus maxima curves. This allows them to follow QRS wave across scales in noisy signals, and better define the spectral region corresponding to the QRS maxima peak. The the algorithm using patient signals recorded in the Coronary Care Unit of the Royal Infirmary of Edinburgh with a positive predictive value of $99.73 \%$ and with the MIT/BIH database obtaining a positive predictive value of 99 .

\section{DYADIC WAVELET TRANSFORM}

Consequently, it should be noted that in their algorithm they compute the DyWT for a priori fixed four scales. However, in our approach a specific spline wavelet, suitable for the analysis of QRS complexes is designed and the scales are chosen adaptively based on the signal. We compute the D WT at two consecutive scales and if necessary, compute the DyWT at one additional scale. Usually, we only need to compute the DyWT at two scales which has a computational advantage as compared. Further, we have been tested their algorithm on the MIT/BIH database, whereas our algorithm was tested on the AHA database. In addition, we have thoroughly compared the performance of our algorithm with other standard techniques using the same database. Also considered that the DyWT-based QRS detector is resists to noise. Even though, it is possible to apply their algorithm to detect other waves such as $\mathrm{P}, \mathrm{T}$. in this project, we have mainly concentrated on detecting $R$ waves since we are interested in estimating the heart. In this project proposed a Spline wavelet for detecting QRS complex which is the transient part in the ECG signal. Hence the detection process is based on "the property that the absolute value of DyWT has localized maxima across several consecutive scales at the instant of the occurrence of transient." $68 \%$.

Let us $\psi$ indicates mother wavelet which is a function with zero mean. The dyadic dilation at level $\mathrm{j}$ is given by

$$
\psi_{2^{\mathrm{j}}(\chi)}=\frac{1}{2^{j}} \psi\left(\frac{x}{2^{j}}\right)
$$

The wavelet transforms of $f(x)$ at the dyadic scale $j$ and at the location $\mathrm{x}$ is defined to be

$$
W_{2^{j}} f(x)=f * \psi_{2^{j}}(x)=\int f(t) \cdot \psi_{2^{j}}(x-t) d t
$$

The dyadic wavelet transform is then the sequence of functions

$$
W f=\left(W_{2^{j}} f(x)\right)_{j \in \mathrm{Z}},
$$


And $\mathrm{W}$ is the dyadic wavelet transform operator.

\section{THE CHARACTERISTICS OF ECG}

The time taken, frequency and amplitude of ECG will be varies for each person. These parameter depend on the voltage, the speed and path of the impulse through the heart's electrical system. The human ECG signals are very weak and in the $\mathrm{mV}$ range. The frequency range is $0.05-100 \mathrm{~Hz}$ and a large amount of the useful information contained in the range of $0.5-45 \mathrm{~Hz}$. The normal value of heart beat lies in the range of $60 \mathrm{beats} / \mathrm{minute}$ to $100 \mathrm{beats} /$ minute. The ECGs' amplitude and intervals have useful information in clinic applications. They are defined by the characteristic points of ECG which are defined as $\mathrm{P}, \mathrm{QRS}$ and $\mathrm{T}$ waves (and sometimes a $\mathrm{U}$ wave). Schematic representation of an ECG signal is shown in Figure 3.1.

The P wave: The first ECG wave of the cardiac cycle is the $\mathrm{P}$ wave which indicates opening of the atria. This is a very small and relatively isoelectric segment following the $\mathrm{P}$ wave. The magnitude of the $\mathrm{P}$ wave is normally low $(50-100 \mu \mathrm{V})$ and $100 \mathrm{~ms}$.

The PR Interval: The PR interval starts with the onset of the P wave and ends at the onset of the $Q$ wave. It indicates the duration of the conduction from the atria to the ventricles. Normal duration is $120 \mathrm{~ms}-200 \mathrm{~ms}$.

The PR Segment: The PR segment begins with the endpoint of the $\mathrm{P}$ wave and ends at the onset of the $\mathrm{Q}$ wave. It represents the duration of the conduction from atrioventricular node, down the bundle of its end through the bundle branches to the muscle. There is no deflection during PR interval.

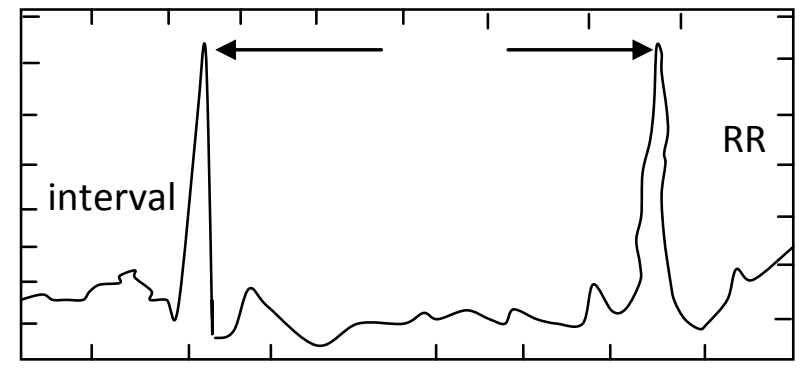

Fig 3.1 A schematic representation of the ECG and its characteristic waves.

The QRS complex: The QRS complex detection due to the period of ventricular contraction or depolarization. It is the result of ventricular depolarization through the Bundle Branches and Parkinje fibre. The QRS complex compared to other wave is a much larger signal due to the volume of depolarization in the left and right sides of the heart move in opposite directions. But either side of the heart chamber is not working properly, then size of the QRS complex may increase.
The ST Segment: The ST segment represents the time between the ventricular depolarization and the depolarization. The ST segment begins at the end of the QRS complex and ends at the beginning of the $\mathrm{T}$ wave. Normally, the ST segment measures 0.12 second or less.

The $\mathrm{T}$ Wave: The $\mathrm{T}$ wave results from the depolarization of the ventricles and is of a longer duration than the QRS complex because the ventricular depolarization happens more slowly than depolarization. Normally, the $\mathrm{T}$ wave has a positive deflection of about $0.5 \mathrm{mV}$, although it may have a negative deflection.

The QT Interval: The QT interval begins at the onset of the Q wave and ends at the end point of the $\mathrm{T}$ wave. It represents the duration of the ventricular depolarization/depolarization cycle

\section{Phases of the Cardiac Cycle}

Atrial Contraction: In this phase of the cardiac cycle which corresponds to the $\mathrm{P}$ wave in the ECG signal as shown in fig.3.2.

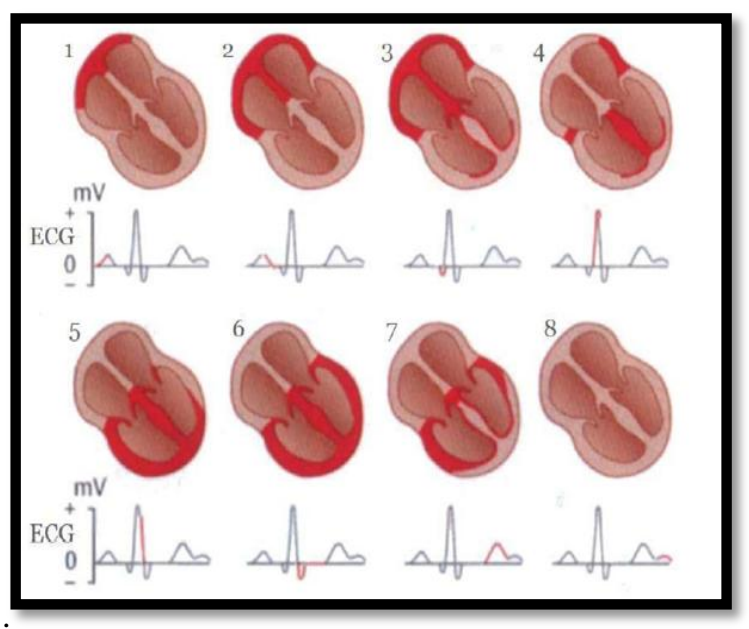

Fig.3.2. Phases and development of the ECG waveform in heart beat

Isvolumetric Contraction: Ventricular depolarisation (the beginning of Ventricular systole), which is visible as the QRS complex in the ECG.

Reduced Ejection: The reduced ejection phase starts with the ventricular repolarisation represented as the $\mathrm{T}$ wave in the ECG.

\section{METHODOLOGY}

The analysis of the method applied to detection of QRS complex. In this paper, we present the selected preprocessing methods, which we consider to be applied to detect QRS based on dyadic wavelet transform in ECG signal. Introductions to 
block diagram of QRS complex, the discrete wavelet transform and some families of mother wavelets are added and at the end the procedure of enhancing ECG is presented. A schematic block diagram of the proposed QRS detecting system is divided into a preprocessing or feature extraction stage including linear and nonlinear filtering and a decision stage including peak detection and decision logic as shown in Fig.4.1.

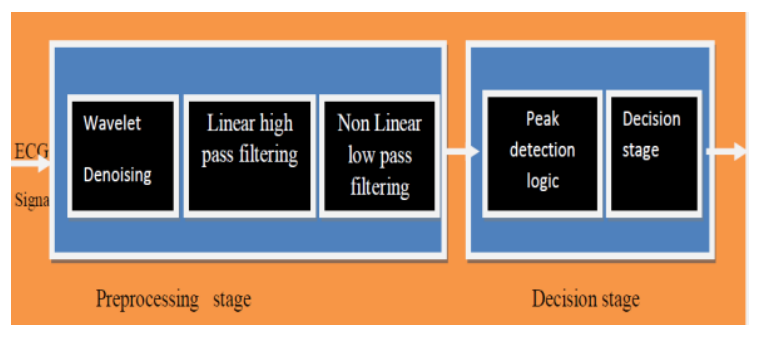

Fig 4.1 Block diagram of QRS detection system.

The task of QRS detection is performed as follows. When ECG signal applied to the system, first preprocessed by wavelet-based denoising in a real-time way to reduce the unstructured noise level. Following the wavelet denoising stage, the denoised signal is then passed into a movingaveraging-based linear HPF to stress the QRS complex and, for the time being, to suppress the low-frequency noise such as $\mathrm{P}$ and $\mathrm{T}$ waves. afterward, in order to guarantee that the highfrequency, low amplitude artifacts can be extra smoothed down to a certain adequately low level while the QRS feature can be well preserved, the output signal of the linear HPF is next processed by a full-wave rectification and non-linear amplification followed by a sliding-window summation, thus resulting in a pulse train-like feature waveform. Together, all the operation, at this stage can be essentially viewed as a nonlinear LPF process. Finally, task of QRS complex detection can be used adaptive thresholding is directly applied and feature waveform to perform decision-making.

\section{Wavelet Transform:}

The transform of a signal is another form of representing the signal. The information content present in the signal does not varying. The Wavelet Transform provides a time-frequency representation of the signal. The disadvantages of short time Fourier transform (STFT) we used wavelet transform, which can also be used to analyze non-stationary signals. While STFT gives a steady resolution at all frequencies, the Wavelet Transform uses multi-resolution technique by which different frequencies are analyzed with different resolutions.

A wave is an oscillating function of time or space and is periodic. In difference, wavelets are localized waves. They have their energy determined in time or space and are suited to analysis of transient signals. While Fourier Transform and STFT use waves to analyze signals, the Wavelet Transform uses wavelets of finite energy

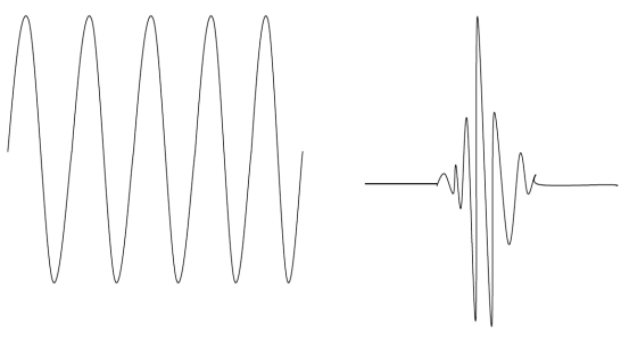

Fig. 4.2 Demonstrations of Wave \& Wavelet

So wavelet analysis is same as the STFT analysis. The signal to be analyzed is multiplied with a wavelet function multiplicand and multiplied with a window function in STFT, and subsequently the transform is calculated for each one segment generated. on the other hand, unlike STFT, in Wavelet Transform, the width of the wavelet function changes with each spectral component. The Wavelet Transform gives fine time resolution at high frequency and poor frequency resolution at low frequencies, the Wavelet Transform gives good frequency resolution and poor time resolution.

\section{Comparison between $D_{\mathbf{y}} W T$ with algorithms}

For the sake of completeness we can compare the performance of $\mathrm{D}_{\mathrm{y}} \mathrm{WT}$ QRS detection with following algorithms okada, suppappola and Sun, Hamilton and Tompkins. These algorithms briefly explained as follows.

\subsection{Okada algorithm}

This algorithm applied to digital filter technique to avoid problem of QRS detector. The difference between 1) a threepoint moving average of the ECG signal and 2) a more severe low-pass filtering of the ECG is computed, which successfully make a band pass filtering operation on the original data, then difference signal is squared. To additional highlight the highfrequency

\subsection{MOBD Algorithm}

This algorithm invented by Suppappola and Sun, referred to as multiplication of the backward difference (MOBD), this used for only nonlinear filtering to detect the QRS complex.

\subsection{Hamilton-Tompkins Algorithm}

Finally QRS detector developed by Hamilton and Tompkins uses an optimized bandpass filter-method. This method applies both linear and nonlinear filtering of the ECG to get the slope, duration, and amplitude information of the QRS complex. A digital bandpass filter restricts the frequency passband from $5-12 \mathrm{~Hz}$ to reduce noise effects. After that, the large slope of the $\mathrm{R}$ wave is improved by differentiating and then squaring the signal. Hence smooth signal produced using a moving window integration to obtain a short-time energy estimate which is then used as a detection statistic. Hence, we 
consider DWT algorithm is theoretically similar to the Hamilton-Tompkins algorithm.

\section{IMPLEMENTATION}

The detection of QRS complex in ECG are based on the same basic structure. A linear low pass filter, followed by some nonlinear transformation which conditions the signal prior to the decision rule. This is also in the case of EGM QRS wave detectors, so generally this is very simple implementations including a bandpass filter and an amplitude threshold. The fig 5.1 shows the flow chart of Wavelet Transform based QRS Detection.

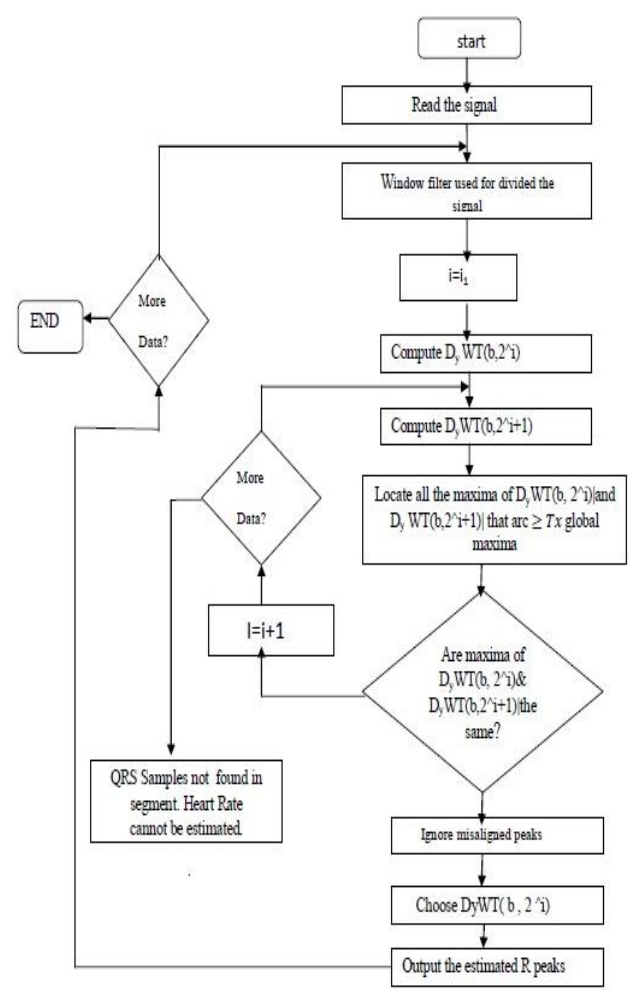

Fig.5.1 Flow chart of Wavelet Transform based QRS Detector

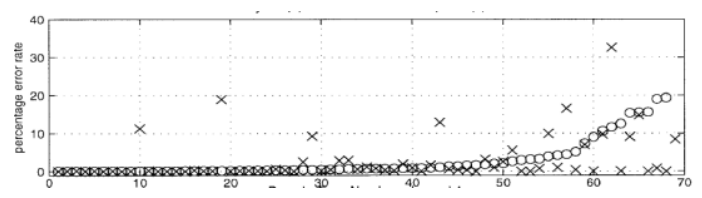

Fig.5.2 Graph of DWT verses Hamilton-Tomkins algorithm

The figure 5.2 shows comparison of the percentage error rate for the DyWT QRS detector versus the Hamilton-Tompkins algorithm. The tapes are sorted in order of non decreasing error rate for the DyWT.

$$
\text { DyWT (0) versus MOBD (*) }
$$

Pseudo-Tape number channel 1

Fig.5.3 Graph of DWT verses MOBD algorithm
The Fig.5.3 shows comparison of the percentage error rate for the DyWT QRS detector versus the MOBD QRS detector algorithm. The tapes are sorted in order of non decreasing error rate for the DyWT.

\section{DyWT (0) versus Okada (*)}

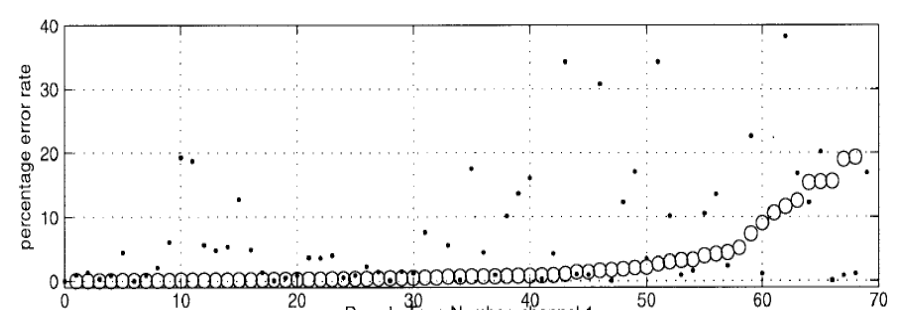

Pseudo-Tape number channel 1

Fig.5.4. Graph of DWT verses Okada algorithm

The fig.5.4 shows comparison of the percentage error rate for the $\mathrm{D}_{\mathrm{y}} \mathrm{WT}$ QRS detector versus the Okada QRS detector algorithm. The tapes are sorted in order of non decreasing error rate for the $\mathrm{D}_{\mathrm{y}} \mathrm{WT}$.

After comparing the percentage of tapes in the AHA database, for each algorithm, that have an overall error rate of less than $1 \%$ and $5 \%$, respectively. Using a $1 \%$ maximum error criterion, for channel 1, the Hamilton-Tompkins algorithm exhibits the best performance $(66 \%$ of the tapes are within the $1 \%$ error criterion while the Okada algorithm exhibits the worst performance (only $27 \%$ of the tapes have an error rate of less than 1\%) in table I . Note that when the error criteria are relaxed (less than 5\%) the DyWT-based QRS detector performs slightly better than the H-T method (for channel 1).

\section{RESULTS AND DISCUSSION}

The output obtained when implementing the algorithm of wavelet transform based QRS detector using different AHA databases in MATLAB software is as shown in Fig.6.1

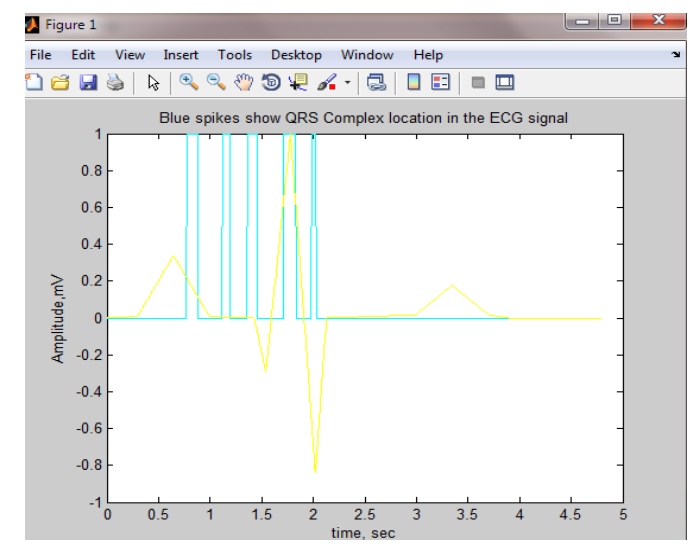

Fig.6.1 Snapshot for detection of QRS complex. 
Fig .6.1 represents the detection of QRS complex in ECG signal using dyadic wavelet transform. Blue spikes shows exact location of QRS complex and yellow spikes indicates structure of QRS complex. Also obtain amplitude of the peak in milli volt at y axix and time occurance of peak in second at $\mathrm{x}$ axis.

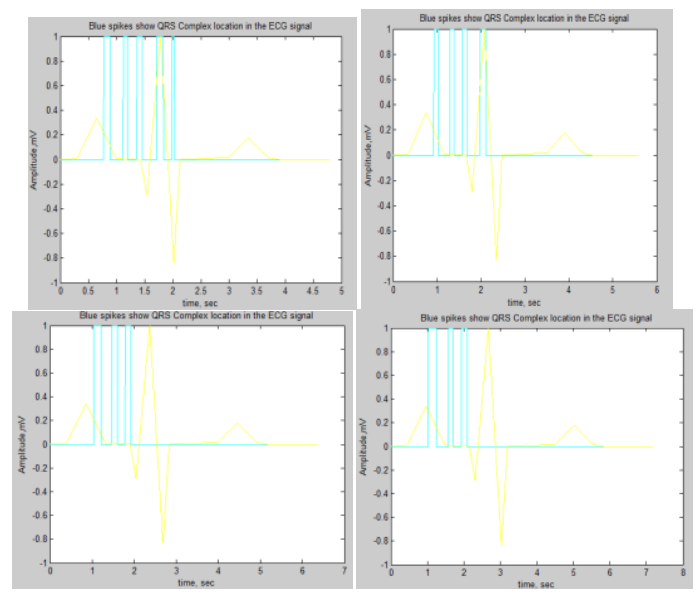

Fig.6.2. a, b, c, and d Snapshots for different QRS complex

Fig.6.2. a,b,c,and $\mathrm{d}$ gives the different location of $\mathrm{R}$ peak detection and different time occurance of $\mathrm{R}$ wave peak because of the number of ECG data changes.

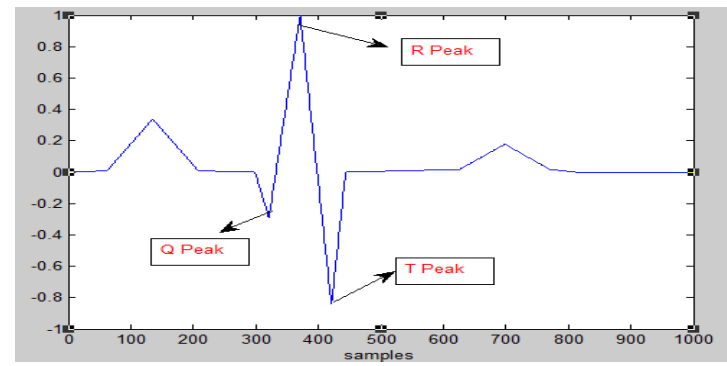

(a)

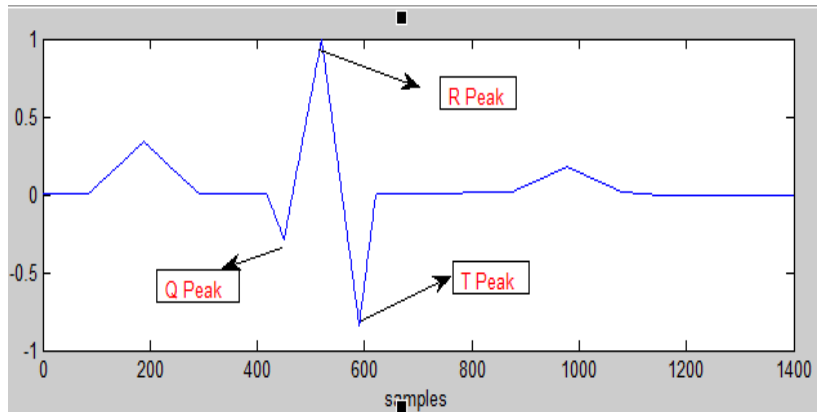

(b)

Fig.6.3. snapshots of exact peak locations of QRS complex
Fig.6.3.The experimental result by matlab code indicates the exact location of Q,R and S in ECG signal. Hence fig.6.3.(a) represented $\mathrm{Q}$ wave peak location at $320, \mathrm{R}$ wave peak location at 381 and $S$ wave peak location at 423 and fig.6.3.(b) represented $\mathrm{Q}$ wave peak location at 435 , $\mathrm{R}$ wave peak location at 510 and $\mathrm{S}$ wave peak location at 594 .

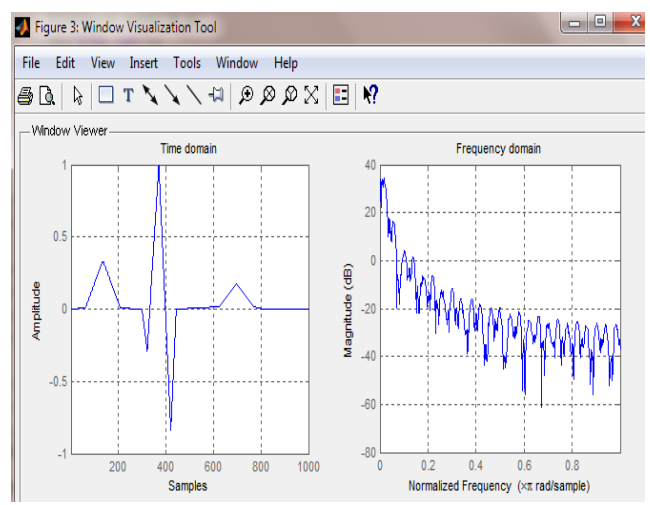

Fig.6.4 snapshot for time domain and frequency domain of QRS

Figure.6.4. characterized time domain and frequency domain of QRS complex detector. By this graph we can identified the maximum peak value of QRS complex detector.

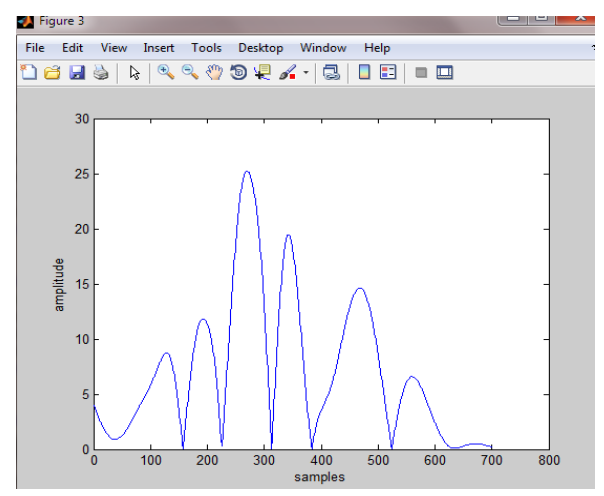

(a)

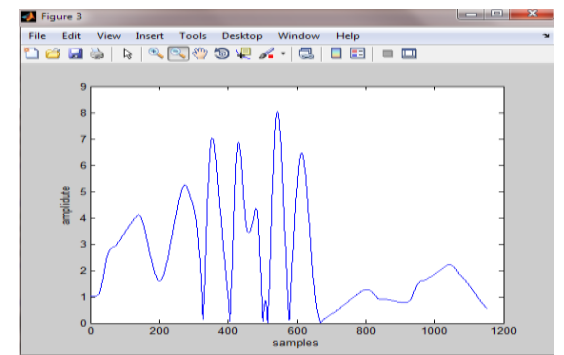

(b)

Fig.6.5 (a) and (b) snapshot for ECG sample 


\section{CONCLUSIONS}

In this paper, a novel algorithm of QRS detection based on DyWT analysis was proposed. The paper exploits the necessary and sufficient properties of DyWT needed to explain ECG signal processing. The property of the onset of the local maxima of the $|\mathrm{DyWT}|$ of a transient signal correlates across successive dyadic scales, particularly when mother wavelet is chosen as the first derivates of the smoothing function. In order to analyze the performance of DyWT based detector is done by testing the algorithm on $70 \mathrm{~h}$ of data standardized AHA database. By performing the feature analysis, the DWT based QRS detection algorithm manifests

Superior performance apart from that for multiform PVC's(3200 tape series), bigeminy (4200 tape series) the couplets (6200 tape series) types the DWT manifested excellent performance .

In this study, a fixed local threshold was used further optimization is not done. The primary advantages of the method can be summarized as below.

1) DWT gives robust noise performance (external noise bursts formed in the multiform PVC's tape series 60$\mathrm{Hz}$ noise and simulated muscle noise )

2) In this analysis of time varying morphology of ECG data the DWT algorithm gives higher range of flexibility.

\section{REFERENCES}

[1]. Wavelet Transform-Based QRS Complex Detector Shubha Kadambe, Member, IEEE, Robin Murray, and G. Faye Boudreaux-Bartels IEEE transactions on biomedical engineering, vol. 46, no. 7, july 1999.

[2]. QRS Complex Detection using Wavelet Transform Rakeshkumar R. Yadav M.E. student (EXTC), D.J. Sanghvi College of Engineering International Journal of Engineering Science and Innovative Technology (IJESIT) Volume 2, Issue 3, May 2013.

[3]. Fetal ECG signal enhancement Maryam Ahmadi, Candidate for the Master of Science in Mechatronics American University of Sharjah, 2008.

[4]. R-peak detection with wavelet transform Asst. Prof. Dr. Sami ARICA Year: 2013, Pages:89 Jury : Asst. Prof. Dr. Sami ARICA: Assoc. Prof. Dr. Zekeriya TÜFEKÇİ: Asst. Prof. Dr. Turgay İBRIKÇİ

[5]. A real-time QRS detection method based on movingaveraging incorporating with wavelet denoising, Szi-Wen Chena,* , Hsiao-Chen Chena, Hsiao-Lung Chanb, 2006

[6]. ECG Feature Extraction Based on Multiresolution Wavelet, TransformS. Z. Mahmoodabadi1,2, A. Ahmadian1,2, M. D. Abolhasani1,2, M. Eslami1, J. H. Bidgoli1,2005

[7]. A New QRS Detection Algorithm Based on the Hilbert Transform, DS Benitez', PA Gaydecki', A Zaidi', AP Fitzpatrick2 0276-6547/00 \$10.00 0 ZOO0 IEEE
[8]. Wavelet based event detection in pacemakers,M., Astrom, S. Olmos, L. S" ornmo Department of Electroscience, Lund University, Lund, Sweden,2001.

[9]. Detection of ECG Characteristic Points UsingWavelet Transforms, Cuiwei Li, Chongxun Zheng, and Changfeng Tai, 1995.

[10]. ECG and APG signal analysis during exercise in a hot environment Charles Darwin University Publication date 2009.

[11]. Application of the Wavelet Transform for Pitch Detection of Speech Signals Shubha Kadambe and G. Faye Boudreaux-Bartels.

[12]. Qrs detection using wavelet transform A thesis submitted in partial fulfillment of the requirements For the degree of Master of Science in Electrical Engineering By Anuja. Nanavati December 2012 